\title{
Research of Fault Detection and Diagnosis for EMB Sensors System Based on Particle Filter
}

\author{
C. Y. Li and Y. N. Xu
}

\begin{abstract}
The electro-mechanical-brake (EMB) system which influences safety and reliability of a pure electric vehicle has received much attention. In EMB system, the feedback sensor signals enhance the accuracy of a pure electric vehicle brake. According to EMB sensor faults, the EMB mathematical model is established based on brushless DC motor (BLDCM). It provides analytical signals for a motor current, a motor speed, and a braking force to an EMB controller. Considering nonlinear characteristics of the EMB system, an observer is designed based on particle filter (PF) algorithm. Then, a sensor state and a fault can be directly estimated by this observer. Also, the residual is used for the sensor faults detection. The simulation result shows that when a sensor has a fault, the designed particle filter not only detects the fault in real-time, but also can locate the fault sensor accurately. The proposed method is proofed feasible and effective.
\end{abstract}

Index Terms-Electro-mechanical brake, fault detection, particle filter, sensor fault.

\section{INTRODUCTION}

With the pursuit of new energy vehicles, it is found that the pure electric vehicle is an ideal system because of its high efficiency, low energy consumption, and low noise. Having considered the safety of a pure electric vehicle, many scholars and automobile manufacturers pay their attention to EMS system. EMB system is a new brake-by-wire system in which the brake power is supplied by an electric motor instead of a traditional hydraulic braking [1]. Main compositions of EMB system include controller, motor, actuator, and other devices. In EMB system, electricity is the unique energy source. The brake command is transferred by electrical signals. And EMB actuator drives the brake plier to clamp brake disc. Then, the wheel brake will be realized.

EMB system adopts a three closed-loop PID control strategy, and the accuracy of vehicle brake is ensured through the feedback sensor signals. A fault sensor may cause an operating error of the controller, even lead to complete failure of EMB system. Therefore, in order to improve reliability of EMB system, problems to be solved have the accurate detection of sensor fault information in real-time, as well as, the selection of the appropriate fault-tolerant control method.

Two independent Kalman filters, effective fault detections of EMB sensors, are constructed in the previous studies

Manuscript received May 2, 2014; revised July 1, 2014. This work was supported by the National Natural Science Foundation of China (61361003) and Jilin University of State Key Laboratory of Automotive Simulation and Control Open Fund (20121120).

The authors are with the division of electronic and communication engineering of Yanbian University, Yanji, China (e-mail: 2013050233@ybu.edu.cn,ynxu*@ybu.edu.cn). published by Rui He [2]. Because the Kalman filter is a linear recursive filter, there is an obvious difference compared to actual nonlinear system after approximating with a linear system. In [3], a mathematical EMB model based on the analytical redundancy method is proposed and the sensor fault detection system is established online. But, this research did not consider the unknown noise which may increase the misdiagnosis rate. In the previous studies, the most of detected faults of sensors are resulted from linear treatments to the nonlinear systems. Therefore, there remain many problems should be solved such as robustness, sensitivity, and practicability of detection algorithm in EMB system.

In order to solve above problems, a nonlinear model is proposed to provide analytic signals for motor current, speed, and braking force sensors in EMB system, respectively. In the model of EMB system, the particle filters based on three kinds of sensor are proposed to realize fault detection in real-time. The simulation result reveals that the sensor fault can be detected in a short time; the proposed method is feasible and effective.

In Section II, the EMB mathematical model is constructed. Section III illustrates the principle of sensor fault detection based on particle filter algorithm. Section IV presents the simulation of sensors fault detection using Matlab. Then conclusions are given in Section V.

\section{EMB MATHEMATICAL MODEL}

EMB system is a new type of brake-by-wire system, thus the EMB actuator is quite different from traditional braking system. The commonly used structure of actuator is made of EMB motor, planetary gear reducer, and ball screw pair. It adopts the planetary gear reducer to improve the output torque of the actuator, and the torque is transformed to translational braking force through the ball screw [4]. The EMB mathematical model is established and analyzed according to the above structure. The structure is shown in Fig. 1.

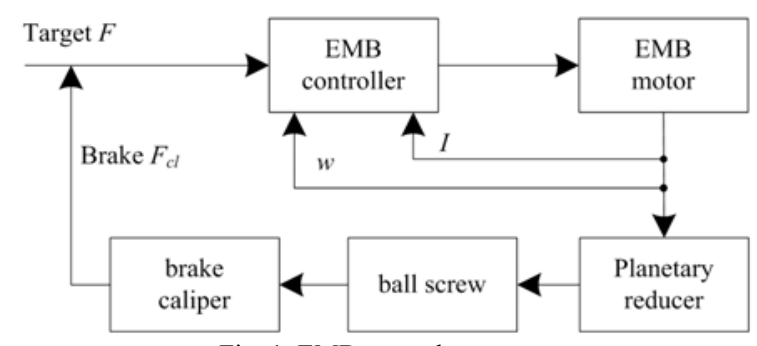

Fig. 1. EMB control structure

When the controller receives the electronic brake signal, it will control the motor operate, then make the actuator output 
the braking force. In order to describe brake intention of the driver accurately and improve the reliability of EMB system, EMB controller uses motor current signal, motor speed signal, and braking force signal to achieve a three closed-loop control.

Through the research of the executive motor model, the analytic model for angular velocity and current signals can be provided. It is assumed that EMB system adopts BLDCM as the executive motor. BLDCM consists of a three-phase stator winding they are a permanent magnetic rotor, an inverter, and other components. According to the dynamic characteristics of BLDCM, electrical and mechanical equations can be established.

Assuming that the three-phase windings are symmetrical, and the sum of three phase currents is zero. Hence, the voltage balance equation of BLDCM can be presented:

$$
\begin{aligned}
{\left[\begin{array}{l}
\boldsymbol{u}_{a} \\
\boldsymbol{u}_{b} \\
\boldsymbol{u}_{c}
\end{array}\right]=} & {\left[\begin{array}{ccc}
L-M & 0 & 0 \\
0 & L-M & 0 \\
0 & 0 & L-M
\end{array}\right] p\left[\begin{array}{l}
i_{a} \\
i_{b} \\
i_{c}
\end{array}\right] } \\
& +\left[\begin{array}{lll}
\boldsymbol{R}_{a} & 0 & 0 \\
0 & \boldsymbol{R}_{b} & 0 \\
0 & 0 & \boldsymbol{R}_{c}
\end{array}\right]\left[\begin{array}{l}
\boldsymbol{i}_{a} \\
\boldsymbol{i}_{b} \\
\boldsymbol{i}_{c}
\end{array}\right]+\left[\begin{array}{l}
\boldsymbol{e}_{a} \\
\boldsymbol{e}_{b} \\
\boldsymbol{e}_{c}
\end{array}\right]
\end{aligned}
$$

where, $\boldsymbol{u}_{\boldsymbol{a}}, \boldsymbol{u}_{\boldsymbol{b}}, \boldsymbol{u}_{\boldsymbol{c}}$ are stator voltages; $\boldsymbol{i}_{\boldsymbol{a}}, \boldsymbol{i}_{\boldsymbol{b}}, \boldsymbol{i}_{\boldsymbol{c}}$ are three phase stator currents; $\boldsymbol{R}_{\boldsymbol{a}}, \boldsymbol{R}_{\boldsymbol{b}}, \boldsymbol{R}_{c}$ are stator resistances; $\boldsymbol{e}_{\boldsymbol{a}}, \boldsymbol{e}_{\boldsymbol{b}}, \boldsymbol{e}_{\boldsymbol{c}}$ are the back-emf; $\boldsymbol{L}, \boldsymbol{M}$ represent the self-inductance and mutual inductance, respectively; $\boldsymbol{p}$ is the differential operator.

The equation (1) can be rewritten as the equation (2) when the effect of mechanical brush commutation on a motor was not considered.

$$
u=L \frac{d i}{d t}+R i+e
$$

The trapezoidal back-emf of BLDCM is:

$$
\boldsymbol{e}=\boldsymbol{K}_{e} \boldsymbol{\omega}
$$

where, $\boldsymbol{i}$ is the armature current; $\boldsymbol{R}$ is the armature resistance; $\boldsymbol{L}$ is the total armature inductance; $\boldsymbol{u}$ is the armature voltage; $\boldsymbol{K}_{\boldsymbol{e}}$ is the back-emf constant and $\boldsymbol{\omega}$ is the angular velocity for the motor.

The electrical model of the BLDCM can be obtained by (2) and (3).

The equations of the mechanical part can be described as:

$$
\begin{gathered}
J \frac{d \omega}{d t}=T_{e}-T_{L}-T_{f} \\
T_{f}=B \omega+K_{c} \operatorname{sign}(\omega) \\
T_{e}=K_{t} i
\end{gathered}
$$

where, $\boldsymbol{T}_{\boldsymbol{e}}$ is the motor output torque; $\boldsymbol{T}_{\boldsymbol{L}}$ is the load torque; $\boldsymbol{T}_{\boldsymbol{f}}$ is the frictional resistance torque; $\boldsymbol{J}$ is the inertia of the rotor and the losses result from friction; $\boldsymbol{K}_{\boldsymbol{f}}$ is the torque constant; $\boldsymbol{B}$ is the viscose friction; and $\boldsymbol{K}_{\boldsymbol{c}}$ is the Coulomb friction constant.

The resulted equivalent circuit structure of EMB actuator can be presented as shown in Fig. 2.

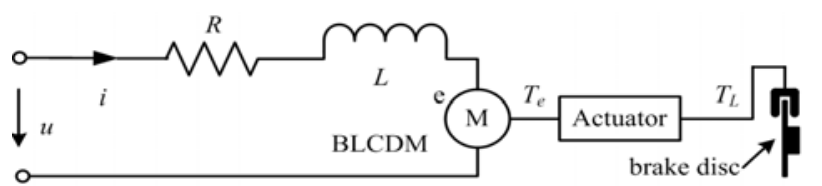

Fig. 2. EMB actuator equivalent circuit structure.

According to (2) - (6), the states of the EMB system can be defined as:

$$
x=\left[\begin{array}{ll}
i & \omega
\end{array}\right]^{T}
$$

Hence, equations of the system state concerned about the motor current $\boldsymbol{i}$ and angular velocity $\boldsymbol{\omega}$ is obtained:

$$
\left\{\begin{array}{l}
\dot{i}=\left(u-R i-K_{e} \omega\right) /(L-M) \\
\dot{\omega}=\left(K_{t} i-B \omega-K_{c} \operatorname{sign}(\omega)-T_{L}\right)
\end{array}\right.
$$

According to the structure of EMB actuator, the relationship between the braking force and the motor load torque can be given by:

$$
F_{c l}=\gamma T_{L}
$$

where, $\boldsymbol{F}_{\boldsymbol{c l}}$ is the braking force; $\boldsymbol{\gamma}$ is the braking force coefficient.

The output of EMB system model can be defined as:

$$
\boldsymbol{y}=\left[\begin{array}{lll}
1 & 0 & 0 \\
0 & 1 & 0 \\
0 & 0 & 1
\end{array}\right]\left[\begin{array}{c}
\boldsymbol{i} \\
\boldsymbol{\omega} \\
\boldsymbol{F}_{c l}
\end{array}\right]
$$

Therefore, the mathematical model of EMB system can be described by (7) - (9).

\section{Sensors Fault Detection and Diagnosis}

Sensor fault is characterized by an obvious deviation between the measured value and the actual value [5]. Fault diagnoses include analytical model-based, signal processing-based techniques, and knowledge-based techniques, mainly [6]. In section II, a relatively accurate mathematics EMB system model has been established. Since, the method based on the analytical model is the preferred one. Given the actual EMB system with nonlinear characteristics, the nonlinear filter is applied for sensor fault diagnosis. Particle filter is a nonlinear estimation method based on signal processing. It abandons the restriction condition that random quantity must satisfy Gaussian distribution when solving the nonlinear filtering problem, and can deal with strongly nonlinear, non-Gaussian random dynamic systems [7]-[8]. Therefore, the particle filter is used as the fault diagnosis method in nonlinear EMB system. Sensor fault diagnosis can be divided into two steps. The first step is to generate residuals which can reflect the sensor fault. The second step is to determine sensor faults according to the 
residual of model.

In EMB system, for monitoring the current, a speed, and braking force signals, three independent particle filters are designed according to (9), respectively. Each filter is only sensitive to one sensor on the purpose of locating the fault sensor. Sensor fault diagnosis structure of EMB system is shown in Fig. 3.

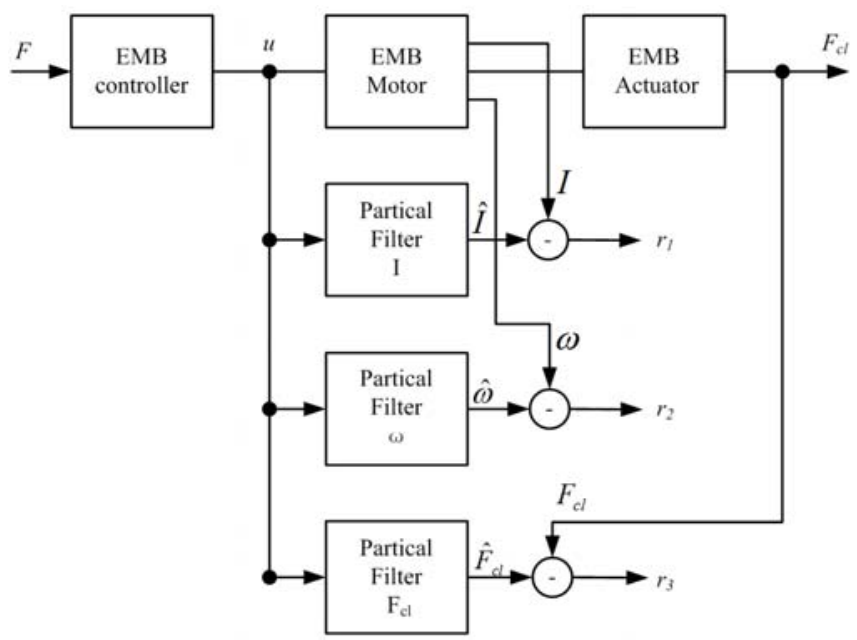

Fig. 3. Sensors fault detection structure of EMB.

In Fig. 3, the controller outputs the control signal $\boldsymbol{u}$ by analyzing the received input target braking force signal $\boldsymbol{F}$. When the control signal $\boldsymbol{u}$ comes, particle filters simulate EMB system and output the estimate motor currents, speeds, and braking force signals. Having compared with measured values from a current sensor, a speed sensor, and a braking force sensor, a fault diagnosis can be implemented. $r_{1}, r_{2}$, and $r_{3}$ represent the residual error, respectively. The definition is:

$$
\begin{aligned}
& \boldsymbol{r}_{1}=\boldsymbol{I}-\hat{\boldsymbol{I}} \\
& \boldsymbol{r}_{2}=\boldsymbol{\omega}-\hat{\boldsymbol{\omega}} \\
& \boldsymbol{r}_{3}=\boldsymbol{F}_{\boldsymbol{c l}}-\hat{\boldsymbol{F}}_{\boldsymbol{c l}}
\end{aligned}
$$

where, $\boldsymbol{I}, \boldsymbol{\omega}$, and $\boldsymbol{F}_{c l}$ denote measured current, speed, and braking force values of sensors, respectively. $\hat{I}, \hat{\omega}$, and $\hat{F}_{c l}$ denote the estimate current, speed, and braking force value obtained by particle filters.

Automobile electronic control and sensor system are always exposed at poor work environment. As a result, they will be affected by outside factors such as humidity, vibration, and electromagnetic fields. In order to improve the reliability of EMB system model, we will consider the influence of outside noise here. Then, the EMB system model proposed in section II can be expressed as follows:

$$
\begin{aligned}
& x_{k+1}=f\left(k, x_{k}, u_{k}\right)+w_{k} \\
& y_{k}=h\left(k, x_{k}\right)+v_{k}
\end{aligned}
$$

where, $\boldsymbol{k}$ represents discrete time; $\boldsymbol{x}_{\boldsymbol{k}}$ is a discrete system state variable; $\boldsymbol{u}_{\boldsymbol{k}}$ is the input control variable; $\boldsymbol{y}_{\boldsymbol{k}}$ is the system output observation; $\boldsymbol{w}_{\boldsymbol{k}}$ and $\boldsymbol{v}_{\boldsymbol{k}}$ denote process noise and observation noise, respectively, which are independent and uncorrelated; $f(\cdot)$ and $h(\cdot)$ are nonlinear functions.

When EMB system is normal, the possible fault characteristics can be described as follows:

$$
\begin{aligned}
& x_{k+1}=f\left(k, x_{k}, u_{k}\right)+w_{k} \\
& y_{k}=h\left(k, x_{k}\right)+g\left(s_{k}\right)+v_{k}
\end{aligned}
$$

where, $\boldsymbol{g}\left(\boldsymbol{s}_{\boldsymbol{k}}\right)$ is a sensor fault function which can be expressed as constant, linear function, nonlinear function, and so on.

Simulated EMB system by particle filters can be expressed as:

$$
\begin{aligned}
& \hat{\boldsymbol{x}}_{\boldsymbol{k}+1 \mid \boldsymbol{k}}=\boldsymbol{f}\left(\boldsymbol{k}, \hat{\boldsymbol{x}}_{\boldsymbol{k} \mid \boldsymbol{k}}, \boldsymbol{u}_{\boldsymbol{k}}\right) \\
& \hat{\boldsymbol{y}}_{\boldsymbol{k} \mid \boldsymbol{k}}=\boldsymbol{h}\left(\boldsymbol{k}, \hat{\boldsymbol{x}}_{\boldsymbol{k} \mid \boldsymbol{k}}\right)
\end{aligned}
$$

where, $\hat{\boldsymbol{x}}_{\boldsymbol{k} \mid \boldsymbol{k}}$ is the optimal estimate of $\boldsymbol{x}_{\boldsymbol{k}}$.

Comparing (12) with (13), assuming that filter has stabilized at $\mathrm{k}$ time, we can get two facts. One is that the system state estimation is equal to the real state. Another is that the state estimation error tends to be zero. Then, get the output residuals:

$$
r_{k}=y_{k}-\hat{y}_{k \mid k}=g\left(s_{k}\right)+v_{k}
$$

When a sensor has fault, $\boldsymbol{E}\left(\boldsymbol{r}_{\boldsymbol{k}}\right) \neq 0$. Otherwise, $\boldsymbol{E}\left(\boldsymbol{r}_{\boldsymbol{k}}\right)$ tends to be zero. Considering the uncertainty of model error, noise interference, etc, an appropriate threshold needs to be set. According to residual values and threshold, the sensor fault can be detected.

\section{The SimUlation ANALYsis}

In this section, based on the research requirement of the particle filter observing method in EMB system, EMB system model will be constructed under Matlab systems in where outputs are motor current, angular velocity, and braking force. Furtherly, the emulation server of the particle filter observer is established, as well as, its arithmetic effectivities and operating possibilities are verified.

TABLE I: Simulation MOdel PARAMETERS IN EMB SySTEM

\begin{tabular}{ll}
\hline \hline Parameter & Value \\
\hline armature inductance, $\boldsymbol{L}(\mathrm{mH})$ & 5 \\
armature resistance, $\boldsymbol{R}(\Omega)$ & 0.5 \\
total motor shaft inertia, $\boldsymbol{J}\left(\mathrm{Kg} \cdot \mathrm{m}^{2}\right)$ & 0.000291 \\
motor damping coefficient, $\boldsymbol{B}(\mathrm{N} \cdot \mathrm{m} \cdot \mathrm{s} / \mathrm{rad})$ & 0.000395 \\
torque coefficient, $\boldsymbol{K}_{\boldsymbol{t}}(\mathrm{N} \cdot \mathrm{m} / \mathrm{A})$ & 0.0697 \\
back-emf coefficient, $\boldsymbol{K}_{\boldsymbol{e}}(\mathrm{V} \cdot \mathrm{s} / \mathrm{rad})$ & 0.0645 \\
\hline \hline
\end{tabular}

To reduce mismatch degrees, EMB system model is designed by adding assumed noises. The parameters of the selected simulation model in EMB system are shown in Table I.

Assuming the system work normally, the sinusoidal wave 
is taken as EMB system input signal, and the rectangular wave is adopted to simulate fault occurred in three kinds of sensors in 1 to $1.5 \mathrm{~s}$, respectively. The simulation results are shown in Fig. 4. The solid line represents the sensor measured values, the point line represents the system output, and the dotted line represents the residual values.
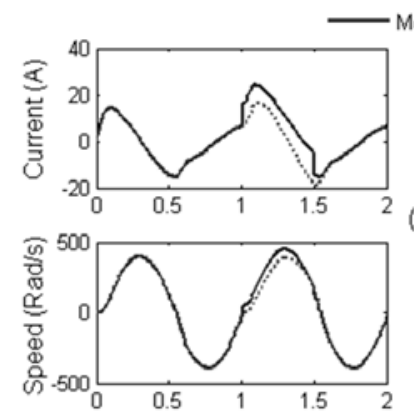

(a)
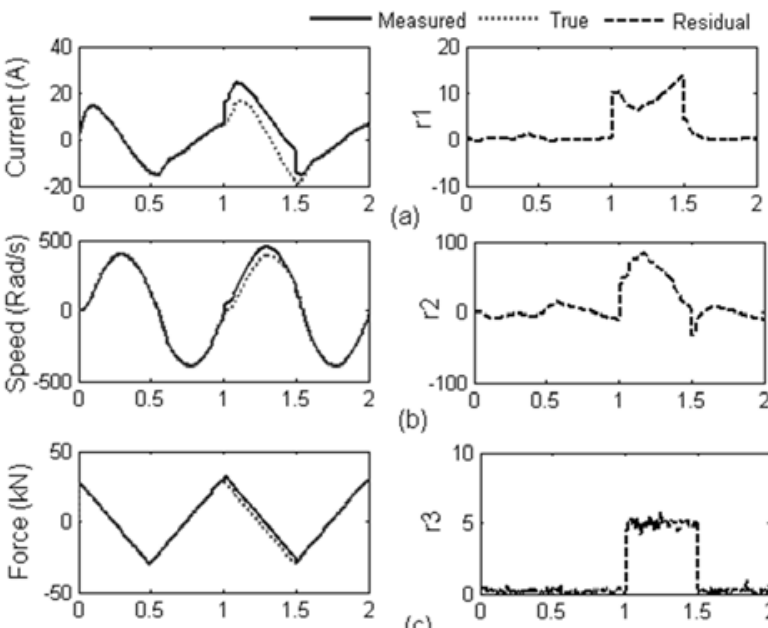

(b)
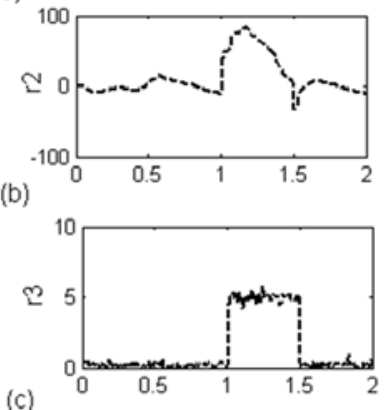

Fig. 4. Sensor fault detection simulation results: (a) current sensor fault detection simulation, (b) speed sensor fault detection simulation, and (c) pressure sensor fault detection simulation.

Fig. 4(a) shows the simulative output current, the measured values of current sensor and the corresponding residual values r1. Fig. 4(b) shows the simulative output speed, the measured values of speed sensor and the residual values r2. Fig. 4(c) shows the output braking force, the measured values of pressure sensors and the residual values r3.

It can be seen from Fig. 4 that in the case of accurate modeling, when a sensor has no fault at 0 to $1 \mathrm{~s}$ and 1.5 to $2 \mathrm{~s}$, there is no difference between the estimated system output from particle filter and the actual measured value. It shows that the state estimation method is accurate and effective. At 1 to $1.5 \mathrm{~s}$, there is an obvious difference between sensors measured value and estimated value, it reflects a sensor has fault. The fault residual curve in Fig. 4 shows that the change is corresponding to the characteristic vector of sensors faults mostly, the fault diagnosis system can provide sensor fault signal precisely in real-time.

\section{CONCLUSION}

In order to achieve the precise braking of vehicles, EMB system adopts three closed-loop feedback control structure, based on the signals of motor current, motor speed and braking force. The feedback signals are obtained by corresponding sensor in EMB system. Sensor faults will cause enormous threat to vehicle safety. With regard to fault detections of a motor current sensor, a motor speed sensor, and a pressure sensor in EMB system, the state estimation model based on the three kinds of sensors is established by particle filter algorithm, respectively. Also, the sensors fault diagnosis system is constructed too. Based on the nonlinear model of EMB system, the robustness and the reliability of fault diagnosis can be improved effectively. And the probability of misdiagnosis is also reduced distinctly. The establishment of particle filters for three kinds of sensors, not only can monitor sensor in real-time to reduce the system cost effectively, but also can achieve the orientation to the fault sensor in order to take effective measure. The simulation results show that the proposed method above is accurate for the sensor fault detection, and each sensor fault detection structure is independent.

\section{REFERENCES}

[1] H. T. D. Ben and K. Durkipp, "Mechatronics and drive-by-wire systems advanced non-contacting position sensors," Elsevier Science Control Engineering Practice, vol. 11, pp. 23, Feb. 2003.

[2] R. He, J. Li, Y. D. Li, C. Huang, and Q. Wei, "Fault detection approach to EMB sensors based on dedicated observers," in Proc. 2011 International Conference on Electric Information and Control Engineering, April 2011, pp. 3266-3269.

[3] W. Hwang, I. Yang, and K. Huh, "Model-based Sensor Fault Detection Algorithm for EMB System," in Proc. 2011 International IEEE Conference on Intelligent Transportation Systems, Oct. 2011, pp. 962-967.

[4] K. Yang, J. Li, Y. D. Li, Z. Rao, S. Tan, and K. Wang, "Development of Electromechanical Brake Actuator and Research of Pressure Estimation," Automobile Technology, pp. 24-26. Oct. 2008

[5] X. Qi, J. Qi, D. Theilliol, Y. Zhang, J. Han, D. Song, and C. S. Hua, “A review on fault diagnosis and fault tolerant control methods for single-rotor aerial vehicles," Journal of Intelligent \& Robotic Systems, vol. 73, pp. 535-555, Jan. 2014.

[6] S. Nandi, H. A. Toliyat, and Li Xiaodong, "Condition monitoring and fault diagnosis of electrical motors-a review," IEEE Trans.on Energy Conversion, vol. 20, pp. 719-729, Dec. 2005.

[7] V. Kadirkamanathan, P. Li, M. H. Jaward, and S. G. Fabri, "A sequential monte carlo filtering approach to fault detection and isolation in nonlinear systems," in Proc. the 39th IEEE Conference on Decision and Control, pp. 4341-4346, vol. 5, Dec. 2000.

[8] F. Daum, "Nonlinear filters: beyond the Kalman filter," IEEE Aerospace and Electronic Systems Magazine, vol. 20, pp. 57-69, Aug. 2005

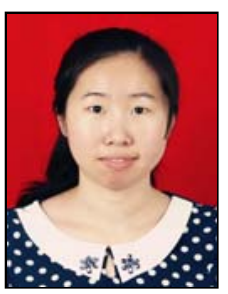

C. Y. Li was born at Jilin Province of China in 1989. She is still pursuing her master degree in Yanbian University, Yanji, China.

She completed bachelor degree electronics engineering and technology, Jilin, Yanji, in the field of electronics and telecommunication, in 2013.

Her research interests include the fault diagnosis and fault-tolerant control.

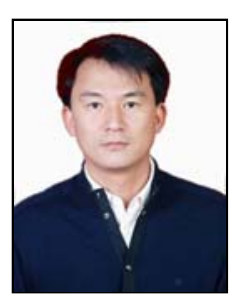

Y. N. Xu was born at Jilin Province of China Currently, he is an associate professor of the division of electronic and communication engineering of Yanbian University, Yanji, China.

$\mathrm{He}$ received the $\mathrm{Ph} . \mathrm{D}$. degree in electrical engineering from the Chonbuk National University, Korea, in 2009. Corresponding author.

His research interests include the in-vehicle networks and automobile electronic control. 